\title{
THE INFLUENCE OF FLEXIBILITY COEFFICIENT ON THE SIZE OF INTERNAL FORCES AND DEFORMATIONS IN CIRCULAR PLATES ON ELASTIC MEDIUM
}

\begin{abstract}
Mirela ȘANDRU - PhD Student, Technical University of Civil Engineering Bucharest, Department of Strength of Materials, Bridges and Tunnels, e-mail: mirela.esandru@yahoo.com

Bogdan Alexandru BUTNARU - PhD Student, Technical University of Civil Engineering Bucharest, Department of Strength of Materials, Bridges and Tunnels, e-mail: bogo_bogdan@yahoo.com

Dumitrel FURIȘ - Professor, PhD, Technical University of Civil Engineering Bucharest

Dan Ilie CREṬU - Professor, PhD, Technical University of Civil Engineering Bucharest, e-mail: cretud@utcb.ro

Abstract: This paper presents an analytical study which deals with the behavior of the circular plates in bending theory, considering the soil-structure interaction under Winkler's hypothesis. It was intended to illustrate the variation of internal forces and deformations according to the flexibility coefficient of plates considering three models: a fixed solid circular plate subjected to a uniformly distributed load, a fixed solid circular plate acted by a displacement applied on the exterior contour and a solid plate subjected to a temperature gradient. For this study the computation relations were written as a product between a dimensional and a non-dimensional factor, the last one indicating the variation of internal forces and deformations. For each type of action there are presented results obtained using the finite element method to illustrate the differences between this method and the analytical computation.
\end{abstract}

Keywords: temperature gradient, stiffness, bending, Winkler medium, foundation

\section{Introduction}

The circular plates - soil interaction is a complex and difficult to handle problem which is encountered in the sizing and the designing of a large number of special, axisymmetric constructions, such as tanks, secondary and primary clarifiers, pumping stations, overhead tank, cement silos, cereals and clinker silos, fermentation tanks and so on. This issue has been and remains one of particular importance, with a large number of scientific papers devoted with solutions which are still far from a satisfactory approximation of the structure -soil interaction.

Theoretical foundation of the plates computation on elastic medium was laid by Winkler in 1867 [1] and later by Boussinesq in 1885 [2], the next developed models appeared in a desire to improve the first two and achieve results closer to real behavior [3], [4], [5].

The structure - soil interaction analysis can be achieved both by using conventional methods available in the literature and also by using software based on finite element method, each having its advantages and disadvantages. Regardless of the method adopted, accuracy of the results is dependent on the computational model and the soil physical and mechanical characteristics of determination.

The purpose of this paper is both to provide an analytical study of the influence of flexibility coefficient on the size of internal forces and deformations due the action of certain loads considering soil a Winkler medium and also to compare results analytically with those provided by the ANSYS Mechanical APDL program.

The Winkler's model was chosen because it is clear and easily to apply, providing satisfactory results for sands and lacking cohesion soils, with a reduced influence of coefficient of subgrade reaction on the results. 


\section{The Winkler's model. State of internal forces and deformations}

The Winkler's model is based on the concept that the soil is a continuous, homogeneous and elastic medium, where the reaction at any point is proportional to the deformation of the point, the feature relation being:

$$
p_{t}(r)=k \cdot w(r)
$$

From a mechanical standpoint, the model is composed of independent springs whose elastic characteristic is the coefficient of subgrade reaction. It is considered that the foundation is in permanent contact with the ground over its all surface, the friction effect in bending study is neglected and the plate deformations are sufficiently small to be allowed to apply the principle of superposition effects.

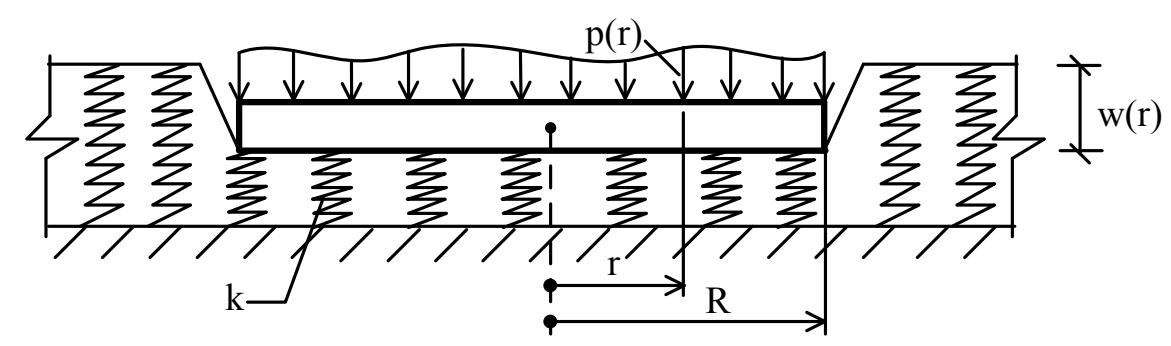

Fig. 1 - Circular plate on Winkler's medium

The state of internal forces and deformations for a circular plate supported on elastic medium as shown on Figure 1, subjected to temperature gradient $\Delta \mathrm{T}$ and to axisymmetric forces applied normaly to plates plane can be studied from the bending plates solution of synthesis equation, together with the boundary conditions. The synthesis equation is a fourth order differential equation, nonhomogeneous with variable coefficients [6], [7]:

$$
\begin{aligned}
& \frac{d^{4} w(\alpha)}{d \alpha^{4}}+\frac{2}{\alpha} \cdot \frac{d^{3} w(\alpha)}{d \alpha^{3}}-\frac{1}{\alpha^{2}} \cdot \frac{d^{2} w(\alpha)}{d \alpha^{2}}+\frac{1}{\alpha^{3}} \cdot \frac{d w(\alpha)}{d \alpha}+w(\alpha)=\frac{p(\alpha) \cdot R^{4}}{D \cdot \lambda^{4}}- \\
& -2 \cdot(1+\mu) \cdot\left(\frac{R}{\lambda}\right)^{2} \cdot \frac{\alpha_{t}}{h} \cdot\left(\frac{d^{2} \Delta T(\alpha)}{d \alpha^{2}}+\frac{1}{\alpha} \cdot \frac{d \Delta T(\alpha)}{d \alpha}\right)
\end{aligned}
$$

In equation (2) the following notations were used:

$$
\begin{aligned}
& \alpha=\rho \cdot \lambda \\
& \rho=\frac{r}{R}
\end{aligned}
$$

$\mathrm{R}=$ radius of circular plate;

$\mathrm{r}=$ radius of a point situated on the circular plate;

$\rho=$ nondimensional variable that defines the position of computational section;

$$
\lambda=R \cdot \sqrt[4]{\frac{k}{D}}
$$

$\lambda=$ the flexibility coefficient of plate;

$\mathrm{k}=$ coefficient of subgrade reaction;

$\mathrm{h}=$ thickness of plate;

$\mu=$ the Poisson's ratio;

$\mathrm{D}=$ flexural stiffness of circular plate; 


$$
D=\frac{E \cdot h^{3}}{12 \cdot\left(1-\mu^{2}\right)}
$$

$\mathrm{E}=$ the modulus of elasticity (Young's modulus);

$\mathrm{w}(\alpha)=$ the normal component of displacement to plate surface, considered the unkown of equation (2);

$\mathrm{p}(\alpha)=$ axisymmetric load aplied normaly to plate surface;

$\Delta \mathrm{T}(\alpha)=\frac{\mathrm{t}_{\mathrm{i}}(\alpha)-\mathrm{t}_{\mathrm{e}}(\alpha)}{2}=$ temperature gradient, $\mathrm{t}_{\mathrm{i}}(\alpha)$ is the bottom face temperature and $\mathrm{t}_{\mathrm{e}}(\alpha)$ is the top face temperature of circular plate.

$\alpha_{\mathrm{t}}=$ thermal coefficient of expansion.

The general solution of syntesis equation $\mathrm{w}(\alpha)$ is composed of a particular solution that depends on the type of load (normal forces to plate surface or temperature gradient) and a solution of the homogeneous equation $\frac{d^{4} w(\alpha)}{d \alpha^{4}}+\frac{2}{\alpha} \cdot \frac{d^{3} w(\alpha)}{d \alpha^{3}}-\frac{1}{\alpha^{2}} \cdot \frac{d^{2} w(\alpha)}{d \alpha^{2}}+\frac{1}{\alpha^{3}} \cdot \frac{d w(\alpha)}{d \alpha}+w(\alpha)=0$ :

$$
w(\alpha)=w_{p}(\alpha)+w_{o}(\alpha) .
$$

The homogeneous solution can be expresed with Bessel functions of first kind, zero order $J_{0}(\alpha)$ and the second kind modified, zero order $K_{0}(\alpha)[6],[7]$ :

$$
w_{o}(\alpha)=A_{1} \cdot J_{0}\left(\alpha i^{-\frac{3}{2}}\right)+A_{2} \cdot K_{0}\left(\alpha i^{-\frac{1}{2}}\right)+A_{3} \cdot J_{0}\left(\alpha i^{\frac{3}{2}}\right)+A_{4} \cdot K_{0}\left(\alpha i^{\frac{1}{2}}\right)
$$

Separating the real part of the solution (8) and using the Kelvin Thomson functions, general solution becomes [6], [7]:

$$
w(\alpha)=w_{p}(\alpha)+C_{1} \cdot \operatorname{ber}(\alpha)+C_{2} \cdot \operatorname{bei}(\alpha)+C_{3} \cdot \operatorname{ker}(\alpha)+C_{4} \cdot \operatorname{kei}(\alpha)
$$

The following notations have been used:

$w_{o}(\alpha)=$ the homogeneous solution of equation (2);

$w_{p}(\alpha)=$ the particular solution of equation (2);

$A_{1}, A_{2}, A_{3}, A_{4}=$ constants of integration;

$C_{1}, C_{2}, C_{3}, C_{4}=$ constants of integration whose values can be obtained due to boundary conditions;

$\operatorname{ber}(\alpha)$, bei $(\alpha), \operatorname{ker}(\alpha), \operatorname{kei}(\alpha)=$ Thomson-Kelvin functions which can be defined with the following relations $[8]$ :

a) with series representation $(\alpha \leq 6)$ :

$$
\begin{aligned}
& \operatorname{ber}(\alpha)=\sum_{k=0}^{\infty} \frac{(-1)^{k} \cdot \alpha^{4 k}}{2^{4 k}[(2 k) !]^{2}} \\
& \operatorname{bei}(\alpha)=\sum_{k=0}^{\infty} \frac{(-1)^{k} \cdot \alpha^{4 k+2}}{2^{4 k+2}[(2 k+1) !]^{2}}
\end{aligned}
$$




$$
\begin{aligned}
& \operatorname{ker}(\alpha)=\left(\ln \frac{2}{\alpha}-C\right) \operatorname{ber}(\alpha)+\frac{\pi}{4} \operatorname{bei}(\alpha)+\sum_{k=1}^{\infty} \frac{(-1)^{k} \cdot \alpha^{4 k}}{2^{4 k}[(2 k) !]^{2}} \sum_{m=1}^{2 k} \frac{1}{m} \\
& k e i(\alpha)=\left(\ln \frac{2}{\alpha}-C\right) \operatorname{bei}(\alpha)-\frac{\pi}{4} \operatorname{ber}(\alpha)+\sum_{k=0}^{\infty} \frac{(-1)^{k} \cdot \alpha^{4 k+2}}{2^{4 k+2}[(2 k+1) !]^{2}} \sum_{m=1}^{2 k+1} \frac{1}{m}
\end{aligned}
$$

where $\mathrm{C}$ is the Euler's constant $C=0.5772 \ldots$.

b) with asymptotic representation $(\alpha>6)$ :

$$
\begin{aligned}
& \operatorname{ber}(\alpha) \cong \frac{e^{\frac{\alpha}{\sqrt{2}}}}{\sqrt{2 \pi \alpha}} \cos \left(\frac{\alpha}{\sqrt{2}}-\frac{\pi}{8}\right) \\
& \operatorname{bei}(\alpha) \cong \frac{e^{\frac{\alpha}{\sqrt{2}}}}{\sqrt{2 \pi \alpha}} \sin \left(\frac{\alpha}{\sqrt{2}}-\frac{\pi}{8}\right) \\
& \operatorname{ker}(\alpha) \cong \sqrt{\frac{\pi}{2 \alpha}} e^{-\frac{\alpha}{\sqrt{2}}} \cos \left(\frac{\alpha}{\sqrt{2}}+\frac{\pi}{8}\right) \\
& \operatorname{kei}(\alpha) \cong-\sqrt{\frac{\pi}{2 \alpha}} e^{-\frac{\alpha}{\sqrt{2}}} \sin \left(\frac{\alpha}{\sqrt{2}}+\frac{\pi}{8}\right)
\end{aligned}
$$

Using equation (9) and considering solid plates resting on elastic medium (constants $C_{3}$ and $C_{4}$ must be zero to have finite deformation in the center of plate) we can express relations for defining the status of internal forces and deformations due to action of axisymmetric forces and temperature gradient:

$$
\begin{aligned}
& w(\alpha)=w_{p}(\alpha)+C_{1} \cdot \operatorname{ber}(\alpha)+C_{2} \cdot \operatorname{bei}(\alpha) \\
& \theta(\alpha)=-\frac{\lambda}{R} \cdot\left[\frac{d w_{p}(\alpha)}{d \alpha}+C_{1} \frac{d b e r(\alpha)}{d \alpha}+C_{2} \frac{d b e i(\alpha)}{d \alpha}\right] \\
& M_{r}(\alpha)=-D \frac{\lambda^{2}}{R^{2}} \cdot\left\{\frac{d^{2} w_{p}(\alpha)}{d \alpha^{2}}+\frac{\mu}{\alpha} \cdot \frac{d w_{p}(\alpha)}{d \alpha}-C_{1}\left[\operatorname{bei}(\alpha)+\frac{1-\mu}{\alpha} \cdot \frac{d b e r(\alpha)}{d \alpha}\right]+\right. \\
& \left.+C_{2}\left[\operatorname{ber}(\alpha)-\frac{1-\mu}{\alpha} \cdot \frac{d b e i(\alpha)}{d \alpha}\right]+2(1+\mu) \frac{R^{2}}{\lambda^{2}} \cdot \frac{\alpha_{t}}{h} \Delta T(\alpha)\right\} \\
& M_{\varphi}(\alpha)=-D \frac{\lambda^{2}}{R^{2}} \cdot\left\{\frac{d^{2} w_{p}(\alpha)}{d \alpha^{2}}+\frac{1}{\alpha} \cdot \frac{d w_{p}(\alpha)}{d \alpha}-C_{1}\left[\mu \cdot b e i(\alpha)-\frac{1-\mu}{\alpha} \cdot \frac{d b e r(\alpha)}{d \alpha}\right]+\right. \\
& \left.+C_{2}\left[\mu \cdot \operatorname{ber}(\alpha)+\frac{1-\mu}{\alpha} \cdot \frac{d b e i(\alpha)}{d \alpha}\right]+2(1+\mu) \frac{R^{2}}{\lambda^{2}} \cdot \frac{\alpha_{t}}{h} \Delta T(\alpha)\right\}
\end{aligned}
$$




$$
\begin{aligned}
& Q_{r}(\alpha)=-D \frac{\lambda^{3}}{R^{3}} \cdot\left[\frac{d^{3} w_{p}(\alpha)}{d \alpha^{3}}+\frac{1}{\alpha} \cdot \frac{d^{2} w_{p}(\alpha)}{d \alpha^{2}}-\frac{1}{\alpha^{2}} \cdot \frac{d w_{p}(\alpha)}{d \alpha}-C_{1} \frac{d b e i(\alpha)}{d \alpha}+\right. \\
& \left.+C_{2} \frac{d b e r(\alpha)}{d \alpha}\right]
\end{aligned}
$$

The following notations have been used:

$\theta(\alpha)=$ rotation of tangent to a deformed fiber in radius direction;

$M_{r}(\alpha)=$ bending moment in radius direction;

$M_{\varphi}(\alpha)=$ bending moment in ring direction;

$Q_{r}(\alpha)=$ shear force.

The positive signs conventions used for internal forces and deformations are shown in Figure 2:

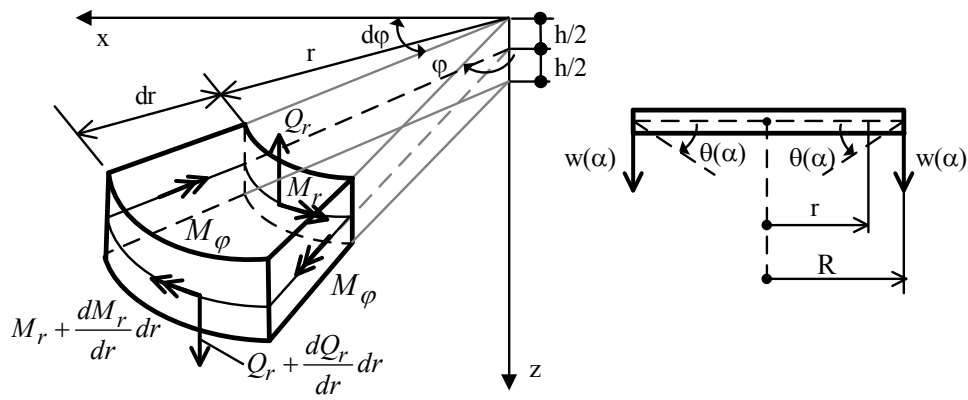

Fig. 2 - The positive signs convention of internal forces on an infinitesimal element and the positive signs convention of deformations for a circular plate

\section{Computational models. The variation of internal forces and deformations due to the flexibility coefficient $\lambda$}

To illustrate the influence of flexibility coefficient on the values of internal forces and deformations three cases were considered:

- $\quad$ 1'st case: fixed solid circular plate subjected to an uniform distributed load p (Fig.3);

- 2'nd case: fixed solid circular plate acted by a displacement $\mathrm{w}_{\mathrm{e}}$ applied on the exterior contour (Fig.4);

- $\quad$ 'rd case: solid circular plate subjected to a temperature gradient $\Delta \mathrm{T}$ (Fig.5).

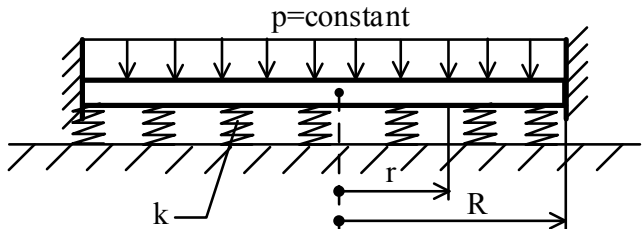

Fig.3 $-1^{\text {st }}$ case

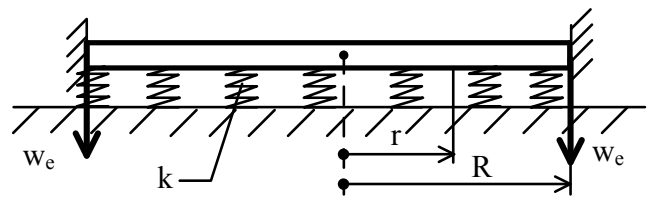

Fig. $4-2^{\text {nd }}$ case

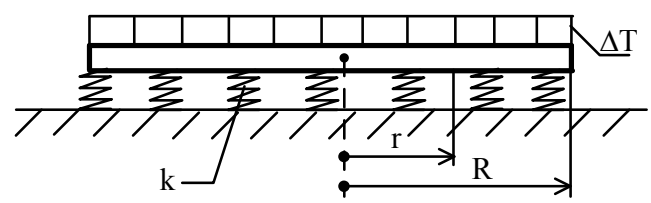

Fig.5 $-3^{\text {rd }}$ case 
Considering the three cases presented, it have been determined the computational relations for internal forces and deformations starting from the general relations (18), (19), ..., (22).

\subsection{Particular solutions, boundary conditions}

The particular solutions for each type of load have been determined considering the form of the right member of equation (2):

- for the first case:

$$
w_{p 1}=\frac{p}{k}
$$

- for the second and the third case:

$$
w_{p 2}=w_{p 3}=0
$$

The integration constants $\left(\mathrm{C}_{1}, \mathrm{C}_{2}\right)$ were determined using the following boundary conditions:

- 1 'st case: for exterior contour $\rho=1 \rightarrow \alpha=\lambda$

$$
\begin{aligned}
& w(\lambda)=0 \\
& \theta(\lambda)=0
\end{aligned}
$$

- 2'nd case: for exterior contour $\rho=1 \rightarrow \alpha=\lambda$

$$
\begin{aligned}
& w(\lambda)=w_{e} \\
& \theta(\lambda)=0
\end{aligned}
$$

- 3'rd case: for exterior contour $\rho=1 \rightarrow \alpha=\lambda$

$$
\begin{aligned}
& M_{r}(\lambda)=0 \\
& Q_{r}(\lambda)=0
\end{aligned}
$$

\subsection{The variation of nondimensional coefficients}

The general computational relations for internal forces and deformations were written as a product of two factors, one nondimensional and another one dimensional; the Poisson's ratio was considered $\mu=0.2$ and a variation between 1 and 15 was chosen for the flexibility coefficient. The nondimensional coefficients $\mathrm{c}(\alpha)$ were plotted depending on the $\rho$ variable.

\subsubsection{Computational relations for the 1'st case}

Computational relations for deformations [7]:

$$
\begin{aligned}
& w_{1}(\alpha)=\frac{p}{k} \cdot c_{w 1}(\alpha) \\
& \theta_{1}(\alpha)=\frac{p}{k \cdot R} \cdot c_{\theta 1}(\alpha)
\end{aligned}
$$




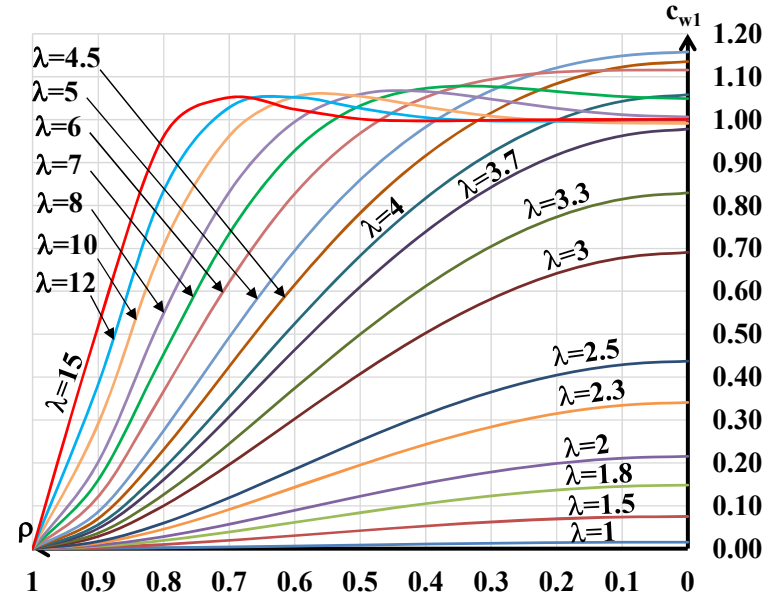

Fig. 6 - Variation of nondimensional coefficient $\mathrm{c}_{\mathrm{w} 1}(\alpha)$

Computational relations for internal forces:

$$
\begin{aligned}
& M_{r 1}(\alpha)=p \cdot R^{2} \cdot c_{M r 1}(\alpha) \\
& M_{\varphi 1}(\alpha)=p \cdot R^{2} \cdot c_{M \varphi 1}(\alpha) \\
& Q_{r 1}(\alpha)=p \cdot R \cdot c_{Q r 1}(\alpha) .
\end{aligned}
$$

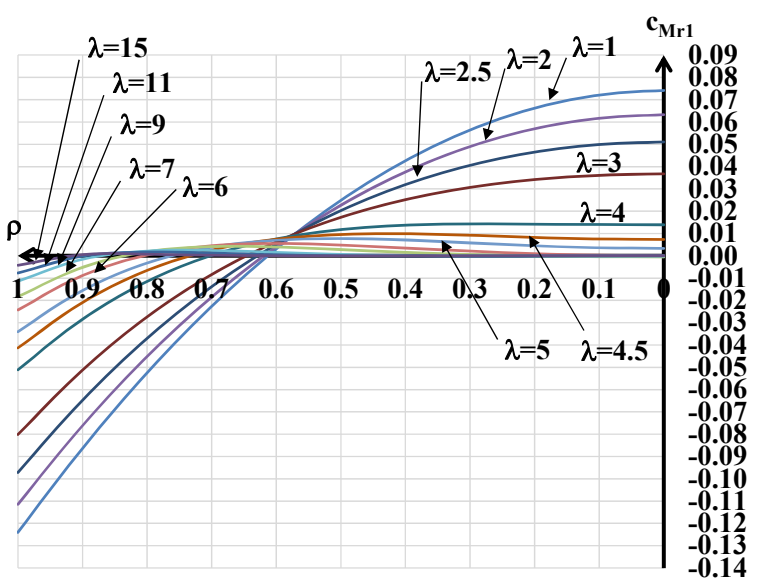

Fig. 8 - Variation of nondimensional coefficient $\mathrm{c}_{\mathrm{Mr} 1}(\alpha)$

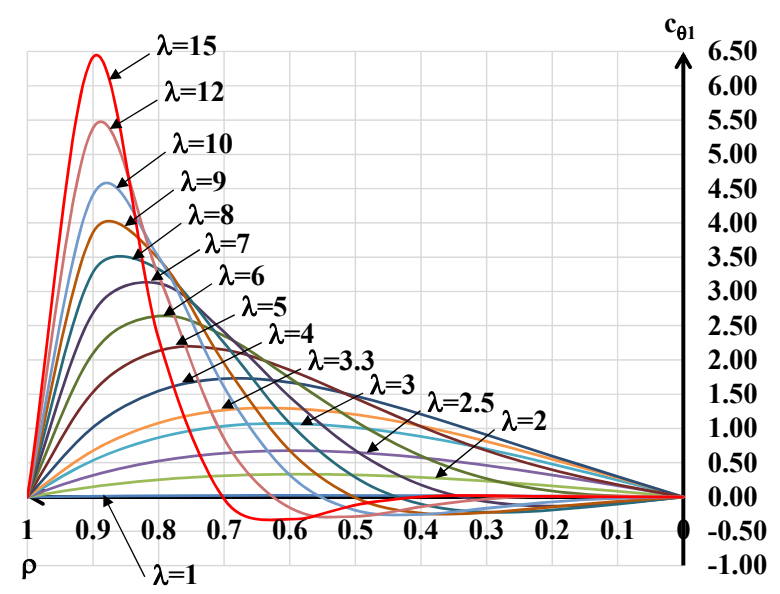

Fig. 7 - Variation of nondimensional coefficient $c_{\theta 1}(\alpha)$

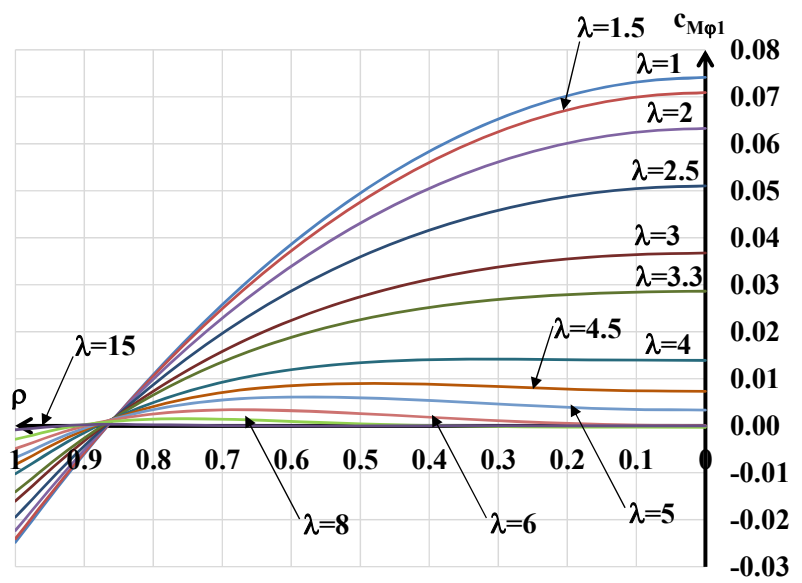

Fig. 9 - Variation of nondimensional coefficient $\mathrm{c}_{\mathrm{M} \varphi 1}(\alpha)$

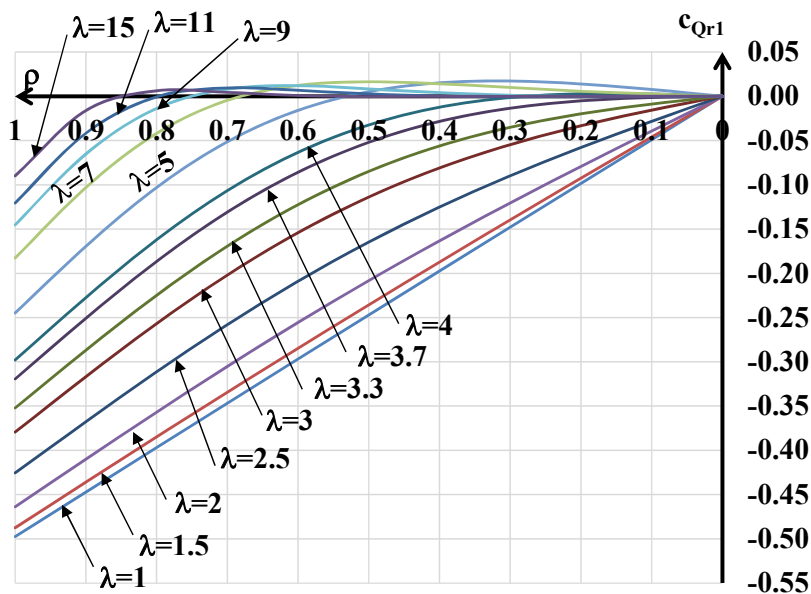

Fig. 10 - Variation of nondimensional coefficient $\mathrm{c}_{\mathrm{Qr1}}(\alpha)$ 


\subsubsection{Computational relations for the 2'nd case}

Computational relations for deformations [7]:

$$
\begin{aligned}
& w_{2}(\alpha)=w_{e} \cdot c_{w 2}(\alpha) \\
& \theta_{2}(\alpha)=\frac{w_{e}}{R} \cdot c_{\theta 2}(\alpha) .
\end{aligned}
$$

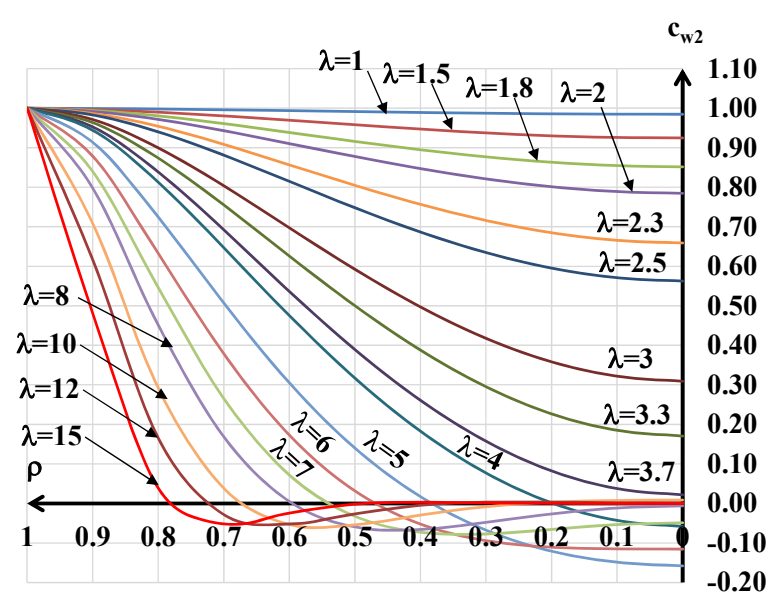

Fig. 11 - Variation of nondimensional coefficient

$$
\mathrm{c}_{\mathrm{w} 2}(\alpha)
$$

Computational relations for internal forces:

$$
\begin{aligned}
& M_{r 2}(\alpha)=w_{e} \cdot R^{2} \cdot k \cdot c_{M r 2}(\alpha) \\
& M_{\varphi 2}(\alpha)=w_{e} \cdot R^{2} \cdot k \cdot c_{M \varphi 2}(\alpha) \\
& Q_{r 2}(\alpha)=w_{e} \cdot R \cdot k \cdot c_{Q r 2}(\alpha) .
\end{aligned}
$$

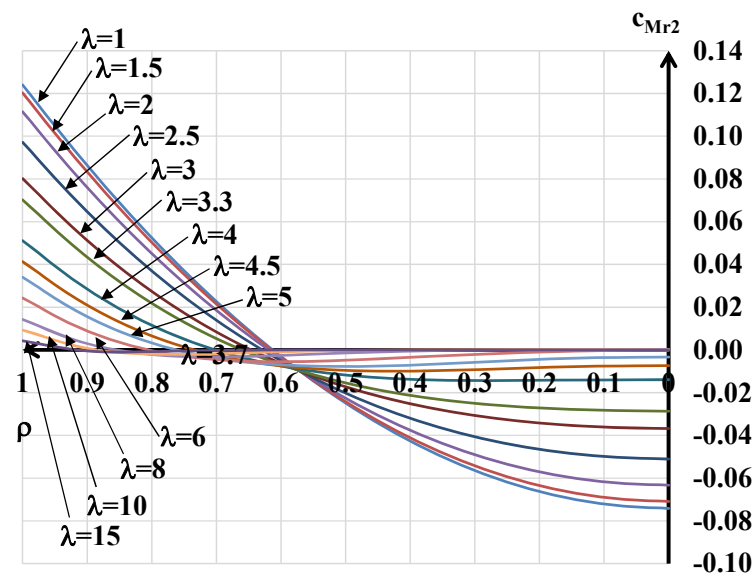

Fig. 13 - Variation of nondimensional coefficient

$$
\mathrm{c}_{\mathrm{Mr} 2}(\alpha)
$$

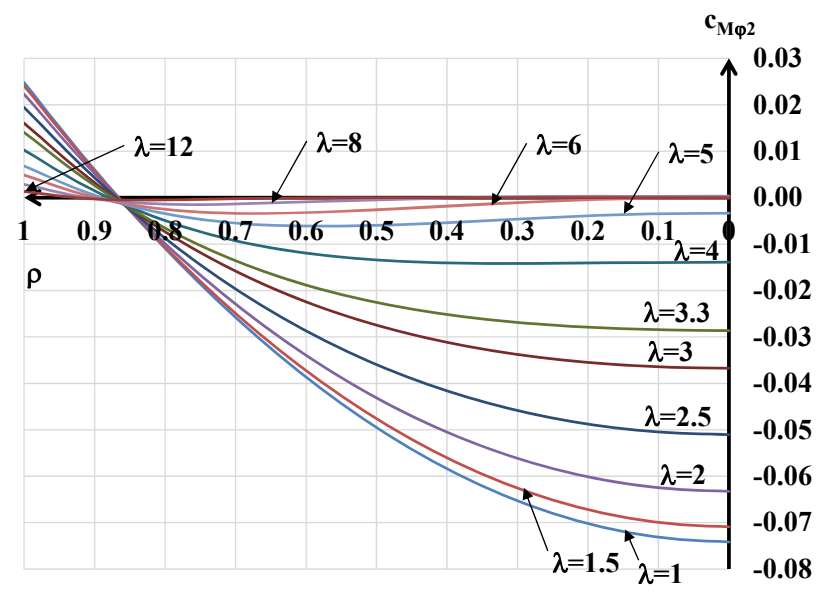

Fig. 14 - Variation of nondimensional coefficient $c_{M \varphi 2}(\alpha)$ 


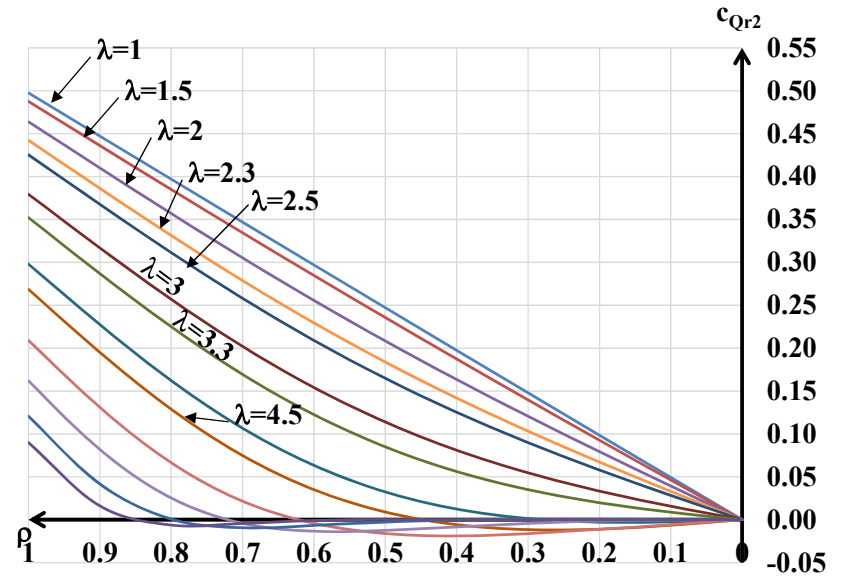

Fig. 15 - Variation of dimensionless coefficient $\mathrm{c}_{\mathrm{Qr} 2}(\alpha)$

\subsubsection{Computational relations for the 3'rd case}

Computational relations for deformations [7]:

$$
\begin{aligned}
& w_{3}(\alpha)=R^{2} \cdot \frac{\alpha_{t}}{h} \cdot \Delta T \cdot c_{w 3}(\alpha) \\
& \theta_{3}(\alpha)=R \cdot \frac{\alpha_{t}}{h} \cdot \Delta T \cdot c_{\theta 3}(\alpha)
\end{aligned}
$$

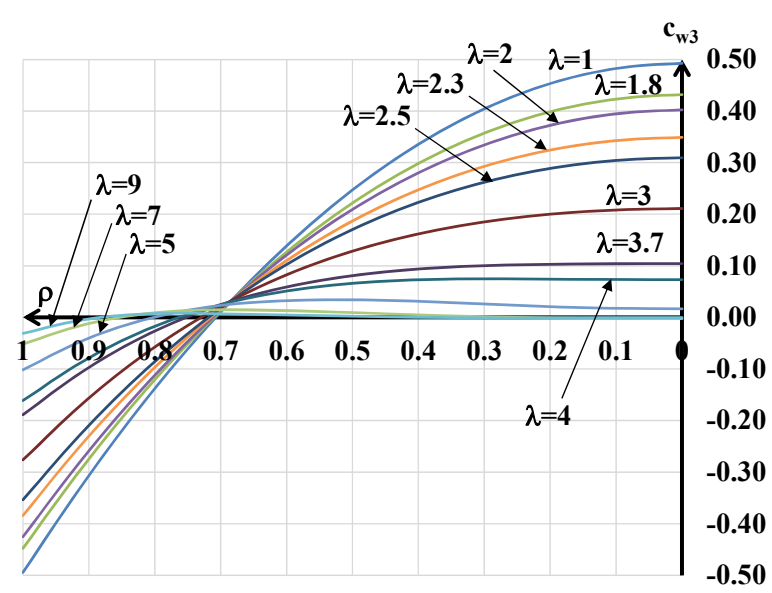

Fig. 16 - Variation of nondimensional coefficient $\mathrm{c}_{\mathrm{w} 3}(\alpha)$

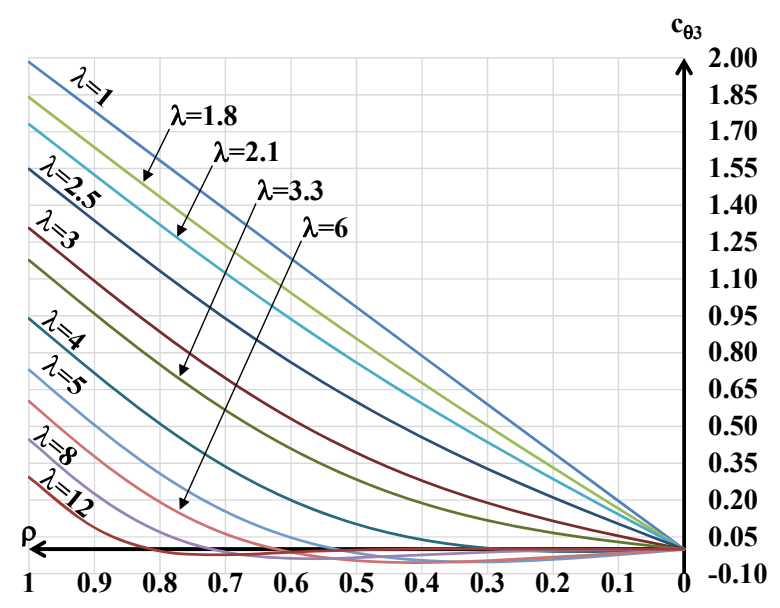

Fig. 17 - Variation of nondimensional coefficient $\mathrm{c}_{\theta 3}(\alpha)$

Computational relations for internal forces:

$$
\begin{aligned}
& M_{r 3}(\alpha)=D \cdot \frac{\alpha_{t}}{h} \cdot \Delta T \cdot c_{M r 3}(\alpha) \\
& M_{\varphi 3}(\alpha)=D \cdot \frac{\alpha_{t}}{h} \cdot \Delta T \cdot c_{M \varphi 3}(\alpha) \\
& Q_{r 3}(\alpha)=\frac{D}{R} \cdot \frac{\alpha_{t}}{h} \cdot \Delta T \cdot c_{Q r 3}(\alpha)
\end{aligned}
$$




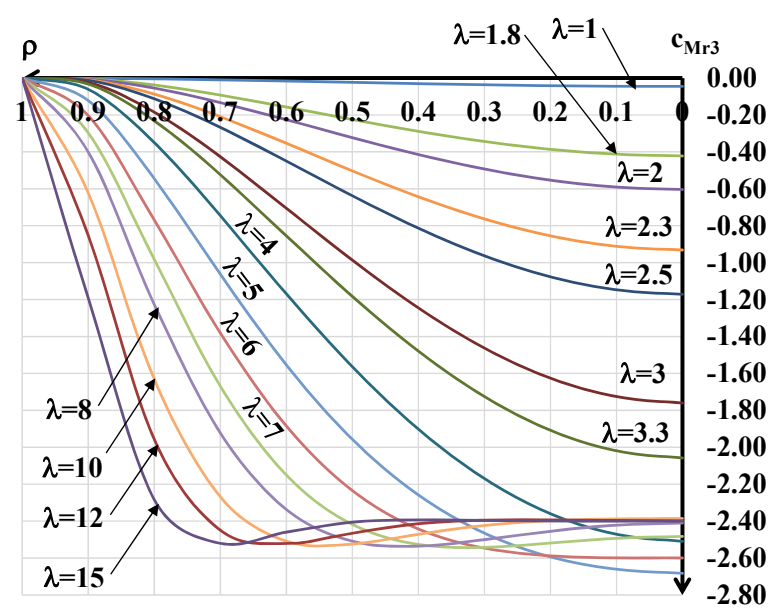

Fig. 18 - Variation of nondimensional coefficient $\mathrm{c}_{\mathrm{Mr} 3}(\alpha)$

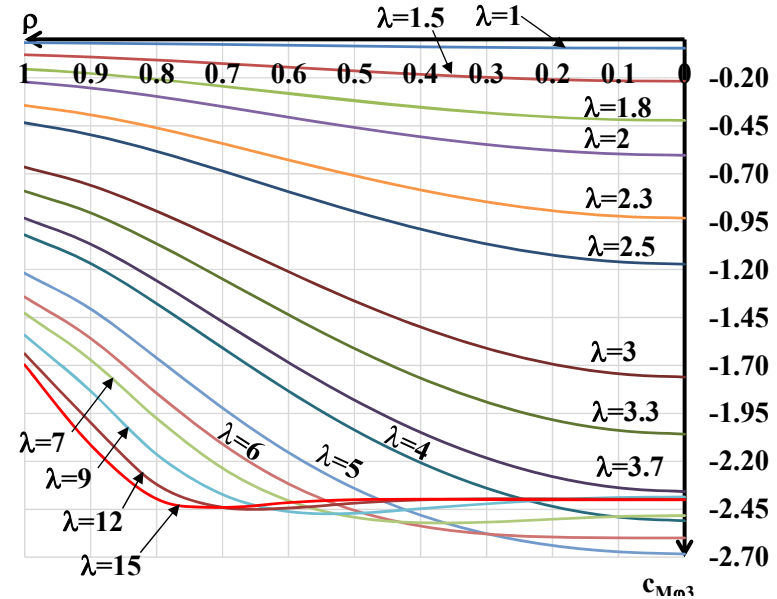

Fig. 19 - Variation of nondimensional coefficient $\mathrm{c}_{\mathrm{M \varphi} 3}(\alpha)$

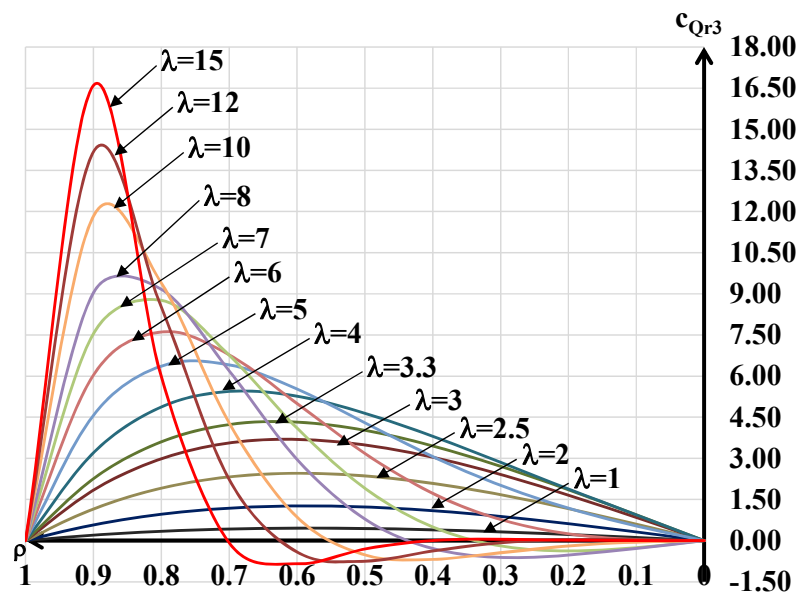

Fig. 20 - Variation of nondimensional coefficient $\mathrm{c}_{\mathrm{Qr} 3}(\alpha)$

\section{Numerical example using the analytical method and the finite element method}

It was considered a circular concrete plate (C20/25 strenght class), Young's modulus E=30 GPa, Poisson's ratio $\mu=0,2$, with the following geometrical characteristics: plate radius $\mathrm{R}=4,50 \mathrm{~m}$, thickness of plate $\mathrm{h}=0,30 \mathrm{~m}$, supported on a Winkler's medium with the coefficient of subgrade reaction $\mathrm{k}=15000 \mathrm{kN} / \mathrm{m}^{3}$. For the three cases presented it was considered:

- $\quad 1$ 'st case: uniformly distributed load $\mathrm{p}=60 \mathrm{kN} / \mathrm{m}^{2}$;

- 2'nd case: displacement $\mathrm{w}_{\mathrm{e}}=0,01 \mathrm{~m}$ applied on the exterior contour;

- $\quad 3$ 'rd case: temperature gradient $\Delta \mathrm{T}=-10{ }^{\circ} \mathrm{C}$.

According to the proposed examples, the value for flexibility coefficient is $\lambda=3,058$.

The internal forces and deformations were determined for each case using the analytical proposed method and ANSYS Mechanical APDL program [9]. For the finite element method (FEM) there have been used SHELL181 elements to define the concrete plate and SURF154 elements for elastic medium. 


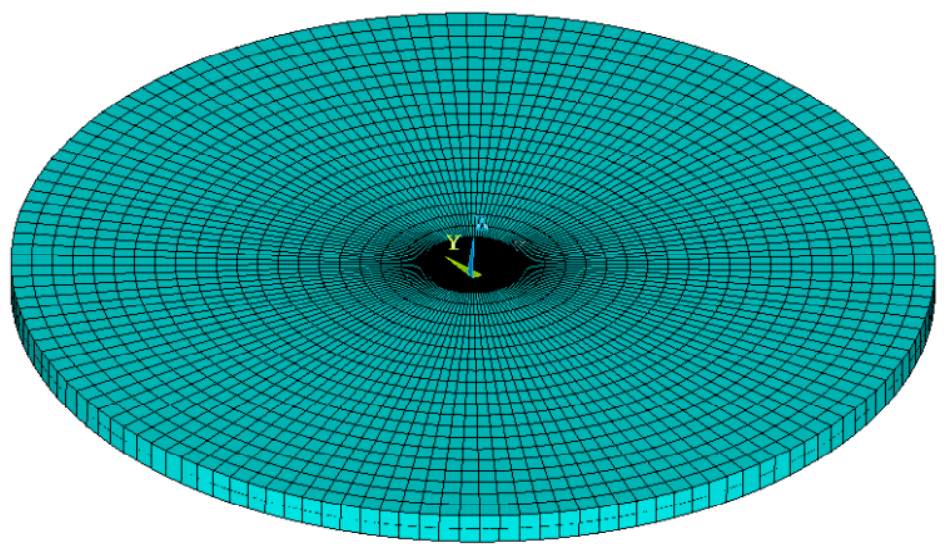

Fig. 21 - The plate mesh - ANSYS Mechanical APDL

The results obtained from the analysis performed are comparatively presented in the following graphs and tables:

Table 1

The variation of deformations $w$ [m] depending on the load type and boundary conditions

\begin{tabular}{|c|c|c|c|c|c|c|}
\hline \multirow{2}{*}{$\rho$} & \multicolumn{2}{|c|}{ 1'st case } & \multicolumn{2}{c|}{ 2'nd case } & \multicolumn{2}{c|}{ 3'rd case } \\
\cline { 2 - 7 } & analytical results & FEM results & analytical results & FEM results & analytical results & FEM results \\
\hline 0 & 0.00288 & 0.00290 & 0.00281 & 0.00275 & -0.00135 & -0.00135 \\
\hline 0.1 & 0.00283 & 0.00285 & 0.00294 & 0.00288 & -0.00134 & -0.00133 \\
\hline 0.2 & 0.00267 & 0.00270 & 0.00332 & 0.00325 & -0.00129 & -0.00128 \\
\hline 0.3 & 0.00243 & 0.00245 & 0.00393 & 0.00387 & -0.00119 & -0.00119 \\
\hline 0.4 & 0.00210 & 0.00212 & 0.00475 & 0.00469 & -0.00105 & -0.00104 \\
\hline 0.5 & 0.00170 & 0.00173 & 0.00574 & 0.00568 & -0.00084 & -0.00083 \\
\hline 0.6 & 0.00127 & 0.00129 & 0.00683 & 0.00677 & -0.00054 & -0.00054 \\
\hline 0.7 & 0.00082 & 0.00085 & 0.00794 & 0.00788 & -0.00015 & -0.00015 \\
\hline 0.8 & 0.00042 & 0.00044 & 0.00895 & 0.00890 & 0.00037 & 0.00037 \\
\hline 0.9 & 0.00012 & 0.00013 & 0.00970 & 0.00966 & 0.00102 & 0.00101 \\
\hline 1 & 0 & 0 & 0.01 & 0.01 & 0.00181 & 0.00180 \\
\hline
\end{tabular}

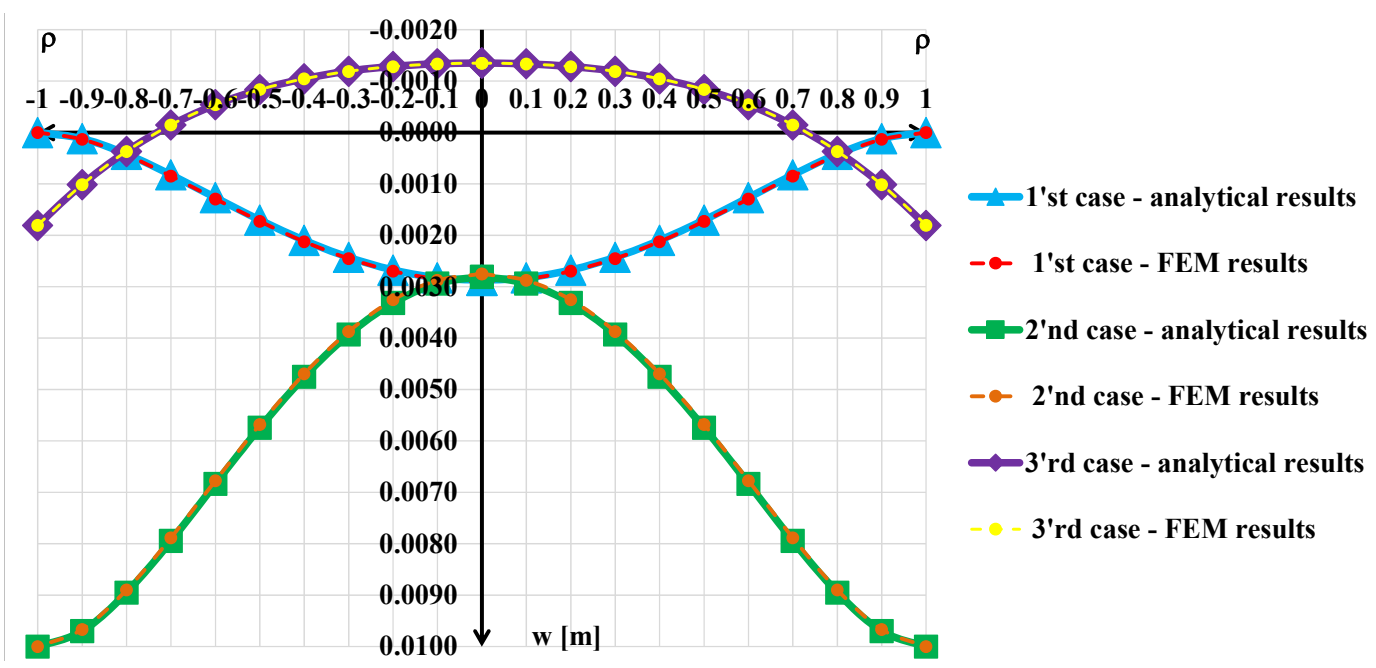

Fig. 22 - The variation of deformations $w$ [m] depending on the load type and boundary conditions 
Table 2

The variation of rotations $\theta$ [rad] depending on the load type and boundary conditions

\begin{tabular}{|c|c|c|c|c|c|c|}
\hline \multirow{2}{*}{$\rho$} & \multicolumn{2}{|c|}{1 'st case } & \multicolumn{2}{c|}{ 2'nd case } & \multicolumn{2}{c}{ 3'rd case } \\
\cline { 2 - 6 } & analytical results & FEM results & analytical results & FEM results & analytical results & FEM results \\
\hline 0 & 0 & 0 & 0 & 0 & 0 \\
\hline 0.1 & 0.00023 & 0.00022 & -0.00057 & -0.00056 & -0.00007 \\
\hline 0.2 & 0.00044 & 0.00044 & -0.00111 & -0.00110 & -0.00016 & -0.00007 \\
\hline 0.3 & 0.00064 & 0.00064 & -0.00161 & -0.00159 & -0.00026 & -0.00026 \\
\hline 0.4 & 0.00081 & 0.00081 & -0.00203 & -0.00201 & -0.00039 \\
\hline 0.5 & 0.00094 & 0.00093 & -0.00234 & -0.00232 & -0.00056 & -0.00039 \\
\hline 0.6 & 0.00099 & 0.00099 & -0.00248 & -0.00246 & -0.00076 & -0.00056 \\
\hline 0.7 & 0.00096 & 0.00095 & -0.00240 & -0.00238 & -0.00100 \\
\hline 0.8 & 0.00081 & 0.00080 & -0.00202 & -0.00199 & -0.00076 \\
\hline 0.9 & 0.00050 & 0.00049 & -0.00125 & -0.00123 & -0.00129 \\
\hline 1 & 0 & 0 & 0 & -0.00160 & -0.00160 \\
\hline
\end{tabular}

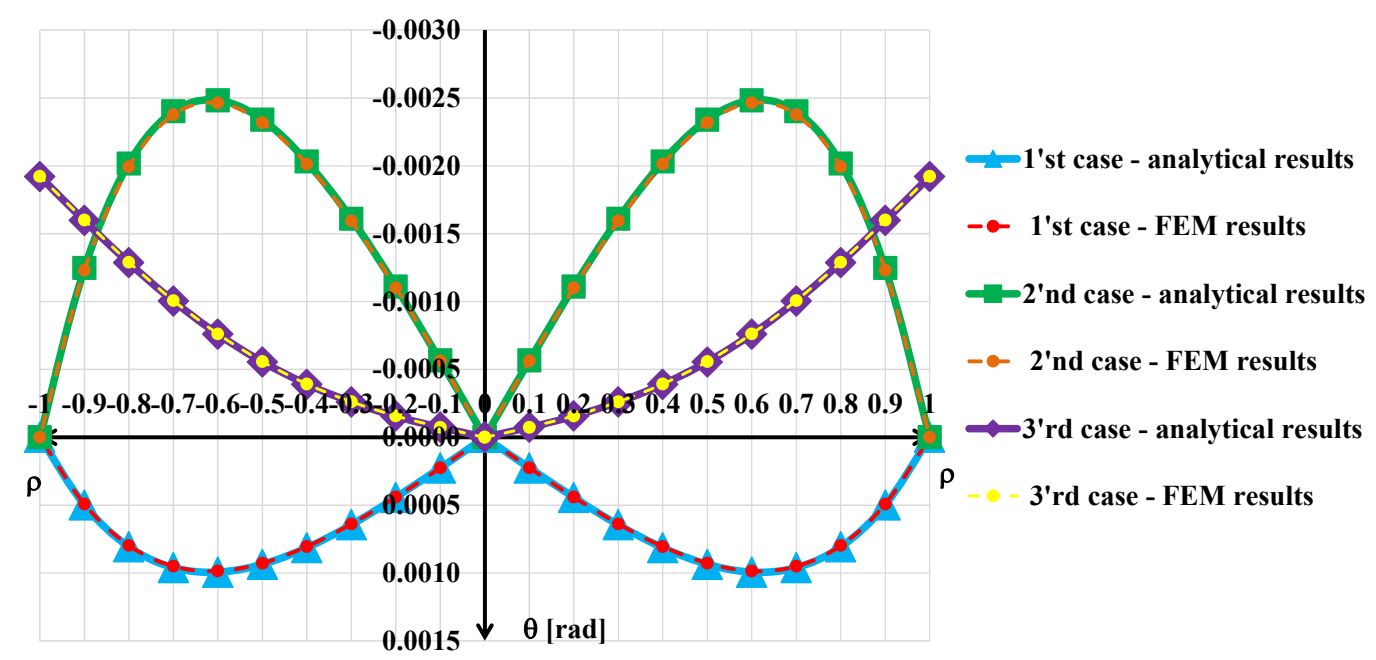

Fig. 23 - The variation of rotations $\theta$ [rad] depending on the load type and boundary conditions

Table 3

The variation of bending moments $\mathrm{Mr}[\mathrm{kNm} / \mathrm{m}]$ depending on the load type and boundary conditions

\begin{tabular}{|c|c|c|c|c|c|c|}
\hline \multirow{2}{*}{$\rho$} & \multicolumn{2}{|c|}{ 1'st case } & \multicolumn{2}{c|}{ 2'nd case } & \multicolumn{2}{c|}{ 3'rd case } \\
\cline { 2 - 7 } & analytical results & FEM results & analytical results & FEM results & analytical results & FEM results \\
\hline 0 & 42.680 & 42.399 & -106.699 & -106.000 & 42.721 & 42.724 \\
\hline 0.1 & 41.989 & 41.724 & -104.971 & -104.310 & 41.901 & 41.909 \\
\hline 0.2 & 39.815 & 39.413 & -99.537 & -98.532 & 39.476 & 39.317 \\
\hline 0.3 & 35.862 & 35.432 & -89.654 & -88.578 & 35.548 & 35.366 \\
\hline 0.4 & 29.649 & 29.227 & -74.123 & -73.067 & 30.308 & 30.142 \\
\hline 0.5 & 20.541 & 20.185 & -51.352 & -50.461 & 24.053 & 23.933 \\
\hline 0.6 & 7.782 & 7.580 & -19.455 & -18.951 & 17.223 & 17.161 \\
\hline 0.7 & -9.442 & -9.622 & 23.604 & 24.054 & 10.436 & 10.451 \\
\hline 0.8 & -31.920 & -31.996 & 79.800 & 79.989 & 4.528 & 4.647 \\
\hline 0.9 & -60.308 & -60.172 & 150.770 & 150.430 & 0.590 & 0.807 \\
\hline 1 & -94.981 & -86.158 & 237.452 & 215.460 & 0.000 & 0.117 \\
\hline
\end{tabular}




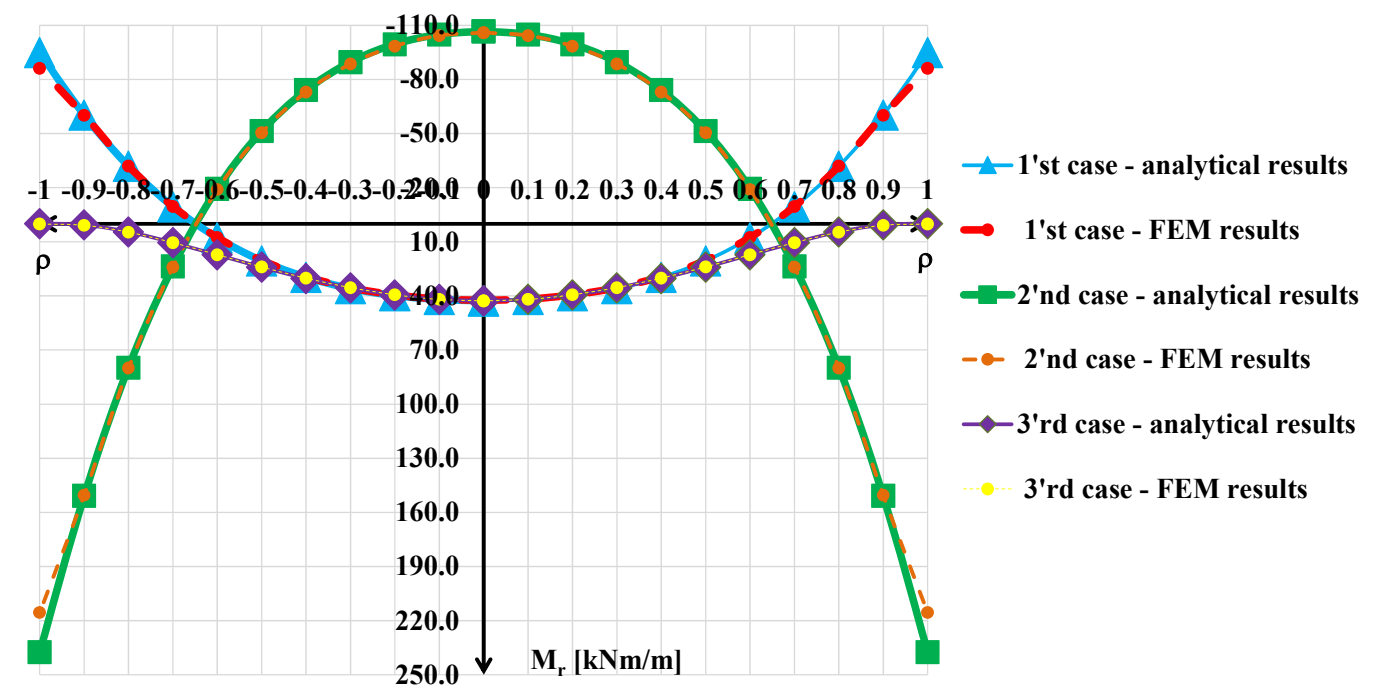

Fig. 24 - The variation of bending moments $\mathrm{M}_{\mathrm{r}}[\mathrm{kNm} / \mathrm{m}]$ depending on the load type and boundary conditions

Table 4

The variation of bending moments $M_{\varphi}[\mathrm{kNm} / \mathrm{m}]$ depending on the load type and boundary conditions

\begin{tabular}{|c|c|c|c|c|c|c|}
\hline \multirow{2}{*}{$\rho$} & \multicolumn{2}{|c|}{ 1'st case } & \multicolumn{2}{c|}{ 2'nd case } & \multicolumn{2}{c|}{ 3'rd case } \\
\cline { 2 - 7 } & analytical results & FEM results & analytical results & FEM results & analytical results & FEM results \\
\hline 0 & 42.680 & 42.399 & -106.699 & -106.000 & 42.721 & 42.725 \\
\hline 0.1 & 42.335 & 42.062 & -105.838 & -105.160 & 42.311 & 42.317 \\
\hline 0.2 & 41.263 & 40.898 & -103.157 & -102.240 & 41.093 & 40.986 \\
\hline 0.3 & 39.348 & 38.956 & -98.370 & -97.387 & 39.108 & 38.969 \\
\hline 0.4 & 36.405 & 36.008 & -91.012 & -90.016 & 36.426 & 36.283 \\
\hline 0.5 & 32.185 & 31.812 & -80.463 & -79.523 & 33.158 & 33.030 \\
\hline 0.6 & 26.389 & 26.080 & -65.973 & -65.188 & 29.467 & 29.363 \\
\hline 0.7 & 18.688 & 18.386 & -46.719 & -45.946 & 25.576 & 25.499 \\
\hline 0.8 & 8.745 & 8.488 & -21.864 & -21.190 & 21.788 & 21.754 \\
\hline 0.9 & -3.741 & -3.905 & 9.351 & 9.820 & 18.494 & 18.506 \\
\hline 1 & -18.996 & -15.329 & 47.490 & 38.376 & 16.189 & 16.584 \\
\hline
\end{tabular}

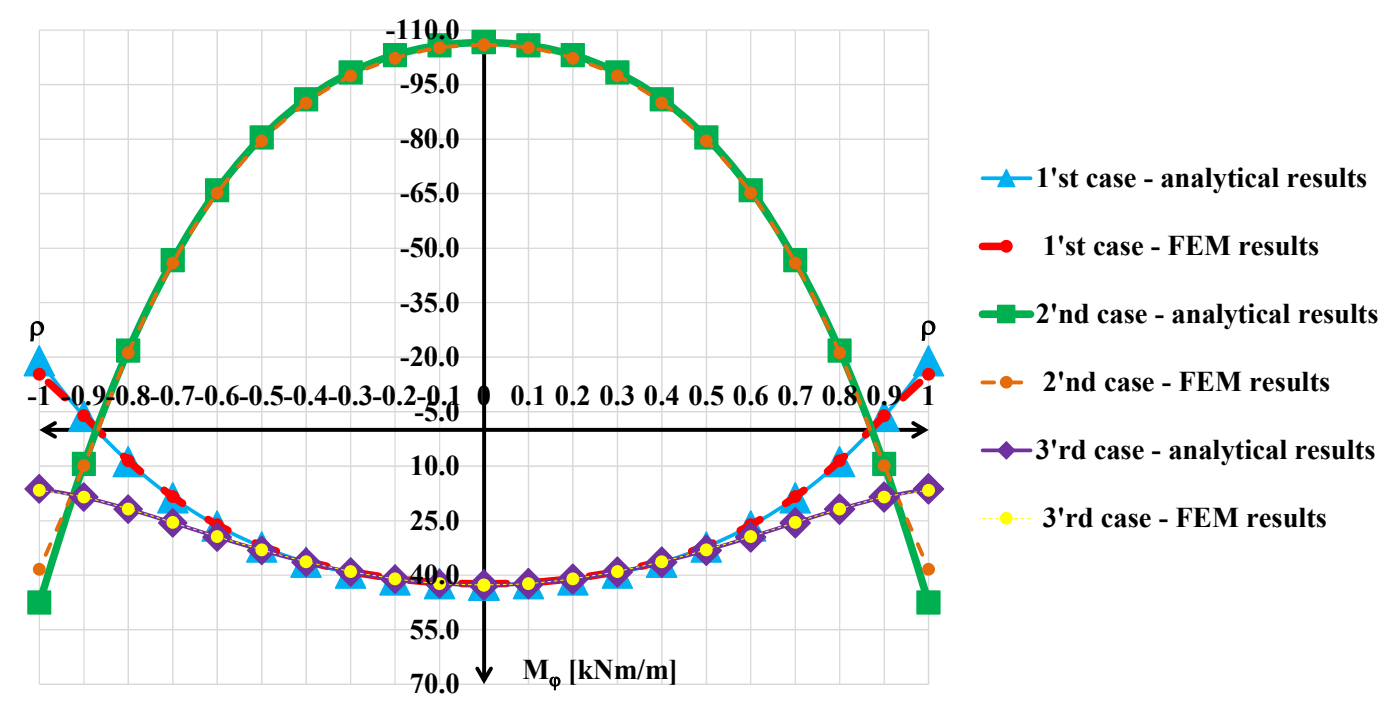

Fig. 25 - The variation of bending moments $\mathrm{M}_{\varphi}[\mathrm{kNm} / \mathrm{m}]$ depending on the load type and boundary conditions 
The variation of shear forces $\mathrm{Qr}[\mathrm{kN} / \mathrm{m}]$ depending on the load type and boundary conditions

\begin{tabular}{|c|c|c|c|c|c|c|}
\hline \multirow{2}{*}{$\rho$} & \multicolumn{2}{|c|}{ 1'st case } & \multicolumn{2}{c|}{ 2'nd case } & \multicolumn{2}{c|}{ 3'rd case } \\
\cline { 2 - 7 } & analytical results & FEM results & analytical results & FEM results & analytical results & FEM results \\
\hline 0 & 0.000 & -0.080 & 0.000 & 0.200 & 0.000 & -0.098 \\
\hline 0.1 & -3.879 & -3.798 & 9.698 & 9.463 & -4.540 & -4.509 \\
\hline 0.2 & -8.271 & -8.187 & 20.678 & 20.446 & -8.909 & -8.891 \\
\hline 0.3 & -13.668 & -13.541 & 34.171 & 33.829 & -12.912 & -12.850 \\
\hline 0.4 & -20.515 & -20.332 & 51.287 & 50.804 & -16.306 & -16.210 \\
\hline 0.5 & -29.177 & -28.909 & 72.942 & 72.244 & -18.779 & -18.661 \\
\hline 0.6 & -39.901 & -39.512 & 99.754 & 98.752 & -19.931 & -19.821 \\
\hline 0.7 & -52.766 & -52.328 & 131.915 & 130.790 & -19.266 & -19.097 \\
\hline 0.8 & -67.611 & -67.093 & 169.028 & 167.720 & -16.190 & -15.977 \\
\hline 0.9 & -83.967 & -83.358 & 209.918 & 208.430 & -10.018 & -9.797 \\
\hline 1 & -100.962 & -96.540 & 252.405 & 241.900 & 0.000 & -2.541 \\
\hline
\end{tabular}

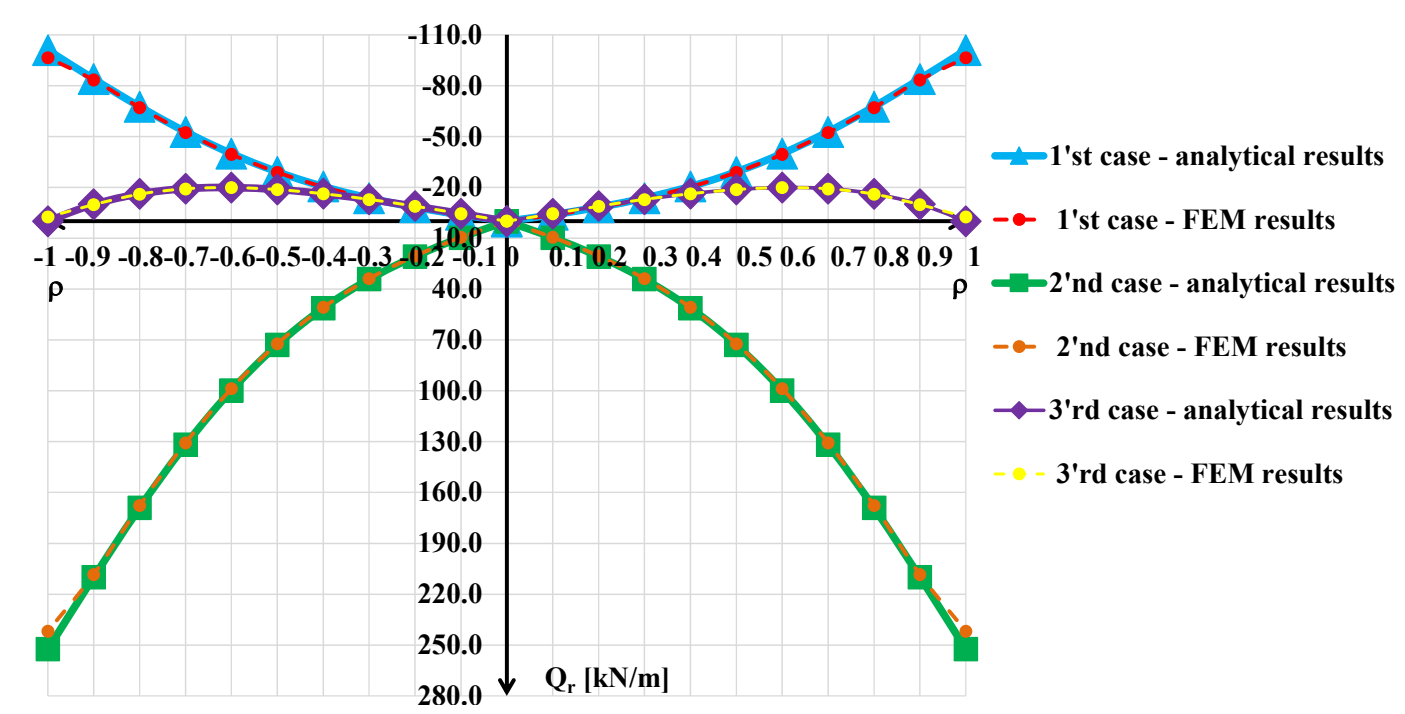

Fig. 26 - The variation of shear forces $\mathrm{Q}_{\mathrm{r}}[\mathrm{kN} / \mathrm{m}]$ depending on the load type and boundary conditions

\section{Conclusions and observations}

Taking into consideration the analyses made in this paper, the state of internal forces and deformations is influenced by the following parameters:

- load type and the functions which define loads;

- boundary conditions for plate;

- the ratio between the plate stiffness and the coefficient of subgrade reaction.

The $\lambda=R \cdot \sqrt[4]{\frac{k}{D}}$ parameter is a flexibility coefficient with which three categories of behavior for plates can be defined:

- $\quad$ rigid plates if $\lambda<1.5$;

- $\quad$ plates with finite flexibility and stiffness if $1.5 \leq \lambda<8$;

- flexible plates if $\lambda \geq 8$.

It was observed that for rigid plates with $\lambda<1.5$, in the first two analysed cases, the contact pressures between plate and soil have a quasi-uniform variation across the plate radius and it 
becomes a parabolic variation with the increase of $\lambda$. The difference between the two cases is that for the first case, the contact pressure increases with the increasing of flexibility coefficient and for the second case the pressure decreases as the plate becomes more flexible, the same phenomenon is encountered for displacement $\mathrm{w}$ and shear force $\mathrm{Q}_{\mathrm{r}}$.

Regarding the rotations, for the first cases the values increase with the increasing of flexibility coefficient, with a parabolic variation with positive or negative values, the variation is changing for flexible plates with maximum values near the exterior contour.

The values for bending moments in radius directions $\mathrm{M}_{\mathrm{r}}$ and in the ring direction $\mathrm{M}_{\varphi}$ (for the first two analysed cases) have high values for rigid plates because the soil response is insignificant and reduced values for flexible plates.

The displacements and the rotations are blocked by soil reaction and consequently the bending moment stretch the cooler fiber of plate in the case of thermal gradient action. The internal forces and deformations depend on flexural stiffness of the circular plate and soil reaction.

For the first two analysed cases the variation of internal forces and deformation is identical to the variation of nondimesional coefficients, but this observation is not available for the third case (for the last case the dimensional coefficient depends on the thickness of plate).

Comparing the analytical results with those obtained from the application of finite element method with the ANSYS Mechanical APDL program there is a good corespondence, the differences being within engineering practice accepted limits (about 3-5\%).

These conclusions and observations are important for the design of structures as knowledge of how the internal forces are located is important for reinforcements. Based on these studies other studies can be achieved considering some new boundary conditions and other types of loads, such that when engineers use programs based on the finite element method they will have control over the results.

\section{References}

[1] Winkler, E. H. (1867). Die Lehre von Elastizität und Festigkeit. Praga, Dominicus.

[2] Boussinesq, J. (1885). Aplication des petentiels à l'étude de l'équilibre et du mouvement des solides elastiques, Paris: Gauthier-Villars.

[3] Sadd, M.H. (2014). Third Edition. Elasticity Theory, Applications, and Numerics, Oxford, Academic Press.

[4] Tsudik, E. (2013). Analysis of Structures on Elastic Foundations, Florida: J. Ross Publishing

[5] Selvadurai, A.P.S. (2013). Vol.17. Elastic analysis of soil-foundation interaction, Amsterdam, Elsevier Scientific Publishing Company.

[6] Timoshenko, S.P., Woinowsky-Krieger, S. (1968) Teoria plăcilor plane și curbe, București, Editura Tehnică

[7] Furiș, D., Erbașu, R., Șandru, M., Butnaru, B.A. (2016), Stări de eforturi și de deformații în plăci circulare, București, Editura Conspress.

[8] Gradshteyn, I.S. Ryzhik, I.M. (2000). Sixth Edition. Table of Integrals, Series, and Products, U.S.A., Academic Press.

[9] ANSYS Mechanical APDL 14.5 Documentation. 